\title{
Incorporation of CdS Nonoparticles from Colloidal Solution into Optically Clear Ureasilicate Matrix with Preservation of Quantum Size Effect
}

\author{
Victor I. Boev ${ }^{\text {a }}$, Alexei Soloviev ${ }^{* b}$, Carlos J. R. Silva ${ }^{\text {a }}$, Maria J. M. Gomes ${ }^{\text {b }}$ \\ ${ }^{a}$ Departamento de Química, Universidade do Minho, 4710-057 Braga, PORTUGAL \\ ${ }^{b}$ Centro de Física, Universidade do Minho, 4710-057 Braga, PORTUGAL
}

\begin{abstract}
Nanocomposite materials based on an organic-inorganic ureasilicate matrix with embedded CdS nanoparticles were produced and characterized by optical (UV/Vis), FTIR, secondary ion mass spectroscopy, inductively-coupled plasma optical emission and steady-state photoluminescence measurements. The ureasilicate precursor was obtained by the reaction between silicon alkoxyde modified by isocyanate groups and polyethylene glycol oligomers with amine terminal groups. The final nanocomposites were prepared by introducing a colloidal solution of $\mathrm{CdS}$ nanoparticles with various sizes into the ureasilicate precursor followed by gelation of the mixture in the presence of ammonia/water vapours. The reliable preservation of the quantum-size effect of nanoparticles after their incorporation into the ureasilicate matrix was observed in all samples. The obtained materials were optically transparent at visible range, exhibiting high flexibility and long-term stability.
\end{abstract}

Keywords: nanocomposites, ureasilicate, $\mathrm{CdS}$, nanoparticles 


\section{Introduction}

The synthesis of semiconductor nanoparticles (NPs) in colloidal solutions has attracted much attention, owing to their unique optical and physical-chemical properties, distinguishing them from the bulk materials. They manifest themselves as, for example, optical nonlinearities ${ }^{1}$, size dependent radiative lifetime ${ }^{2}$ and melting temperature ${ }^{3}$. However, colloidal solutions of NPs are not convenient for producing optically-active devices for optoelectronics. A very important technological task, which can greatly expand the application range of NPs based materials, is the incorporation and immobilisation of NPs in a solid matrix. The unique properties of semiconductor NPs doped-glasses open great prospects for their potential applications in the fabrication of devices for non-linear optics, for optical data storage and for optical switching. ${ }^{4,5}$

Different approaches have been developed in order to obtain these materials. Semiconductor NPs were grown by the gas-diffusion method in a confine media like polymers, ${ }^{6-8}$ zeolites $^{9}$ or sol-gel-derived glasses. ${ }^{10-16}$ In this case, the reaction of a metal-containing solid (or liquid) precursor with a gaseous anion precursor or with some organic molecules, serving as a source of chalcogenide, was used. An additional stabilization of the NPs during their formation was ensured by bifunctional alkoxides and allowed their incorporation into the organic-inorganic matrix. ${ }^{17}$ Radical polymerisation reaction was successfully applied for fabrication of highly luminescent quantum dots/polymer nanocomposites ${ }^{18}$ or polymer matrices containing semiconductor nanocrystals attached to the surface of submicron $\mathrm{SiO}_{2}$ particles. ${ }^{19}$ The ureasilicate matrix doped with CdS particles with the size about $20 \mathrm{~nm}$ was produced by homogeneous precipitation from aqueous solution of $\mathrm{Cd}\left(\mathrm{NO}_{3}\right)_{2}$ and $\mathrm{CH}_{3} \mathrm{CSNH}_{2}{ }^{20}$ However, no evidence of size quantization effect was observed in this work, because of a relatively big size of the particles. 
A promising way to produce nanocomposites is to combine the colloidal chemistry and the sol-gel techniques. It offers better reproducibility and allows flexible control over size, shape and concentration of NPs in the final materials. This procedure has been used for obtaining the optically-clear silica organic-inorganic or pure inorganic nanocomposites, preserving the quantum-size effect of the NPs ${ }^{21-23}$. Generally, it consisted in an addition of semiconductor colloid dispersions, functionalized by amino or mercapto groups, into a separately-prepared pre-hydrolyzed sol followed by gelation and drying of the mixture. In a particular case the functionalized alkoxide was preliminarily added to the matrix precursors ${ }^{24}$. The functional groups act as NP stabilizers hampering their phase separation from the matrix by grafting the NPs to the network of the host material. An alternative approach consists in addition of semiconductor NPs, preliminarily coated with silica shells ${ }^{25}$ or stabilized by TOP/TOPO molecules $^{26}$, into alkoxide precursors.

In the present work we have developed a synthesis procedure for obtaining a flexible and transparent ureasilicate matrix doped with CdS NPs with diameter of 2-3 $\mathrm{nm}$. The preparation procedure permitted us to avoid NPs aggregation without using functional alkoxides. The obtained xerogels were homogeneous and free of cracks. Their optical properties showed the reliable preservation the NPs quantum size effect.

\section{Experimental}

\section{Chemicals}

3-isocyanatepropyltriethoxysilane (ICPTES), tetraethoxysilane (TEOS), dioctyl sodium sulfosuccinate (AOT), 4-fluorothiophenol (FPhSH), and sodium sulphide nonahydrate $(99,99+\%)$ were purchased from Aldrich and used without further purification. O,O'-

bis(2-aminopropyl)-polypropylene glycol-block-polyethylene glycol-block- 
polypropylene glycol -500 (Jeffamine ED-600, Fluka) was dried under a dynamic vacuum before use. Tetrahydrofuran (THF, Merck), isooctane (Fluka), cadmium nitrate tetrahydrate (extra pure, Riedel-deHaën) and a concentrated ammonia solution (25\% w/w, Pronalab) were used as received. High purity water with a resistance around 18 $\mathrm{MS}^{-1} \mathrm{~cm}^{-1}$ was used for the preparation of water solutions of inorganic salts.

\section{Preparation of CdS NPs}

CdS NPs colloidal solutions were produced by using the reverse micelle technique close to the protocol, reported by Shiojiri et al. ${ }^{27}$ Two solutions of AOT in isooctane $(0.1 \mathrm{M}$, $50 \mathrm{~mL})$ were prepared. The stock aqueous solution of $\mathrm{Cd}\left(\mathrm{NO}_{3}\right)_{2} .4 \mathrm{H}_{2} \mathrm{O}(0.13 \mathrm{M}, 0.36$ $\mathrm{mL}$ ) was injected into the first solution and the stock aqueous solution of $\mathrm{Na}_{2} \mathrm{~S}_{9} 9 \mathrm{H}_{2} \mathrm{O}$ $(0.117 \mathrm{M}, 0.36 \mathrm{~mL})$ into the second one. The concentrations of cadmium and sulphur ions in reverse micellar solutions were $0.94 \mathrm{mM}$ and $0.84 \mathrm{mM}$ respectively, providing the ratio $\left[\mathrm{S}^{2-}\right] /\left[\mathrm{Cd}^{2+}\right]=0.9$. Than, the micellar solutions were mixed together and allowed to stand for 3 minutes. The formation of the CdS NPs took place by the exchange of the micelle contents in the collision process, leading to the chemical reaction in the water-pool nanoreactors.

The size of the particles was controlled by changing the molar ratio $w=$ $\left[\mathrm{H}_{2} \mathrm{O}\right] /[\mathrm{AOT}]$, where $\left[\mathrm{H}_{2} \mathrm{O}\right]$ and $[\mathrm{AOT}]$ are the molar concentrations in isooctane of water and AOT respectively. In the experimental conditions described above, $w$ is equal to 4. Increasing the volumes of $\mathrm{Cd}\left(\mathrm{NO}_{3}\right)_{2} \cdot 4 \mathrm{H}_{2} \mathrm{O}$ and $\mathrm{Na}_{2} \mathrm{~S} .9 \mathrm{H}_{2} \mathrm{O}$ to $0.54 \mathrm{~mL}$ and 0.72 $\mathrm{mL}$ gave the $w$ values equal to 6 and 8 respectively. When changing $w$, the concentrations of the cadmium and sulphur in stock solutions were adjusted in order to get the same concentrations in the reverse micellar solutions, as in the case of $w=4$. 
Addition of $100 \mu \mathrm{L}$ of 4-fluorothiophenol to the mixture resulted in flocculation and precipitation of the particles. The pale yellow precipitate was separated by centrifugation. A new portion of isooctane was added to the precipitate and intensively shaken. Then the obtained suspension was centrifuged again. This cycle was repeated 10 times and the final wet precipitate was dissolved in $2 \mathrm{~mL}$ of THF. This procedure was performed in order to remove the most of the AOT from CdS NPs, because the excess of AOT in the final material resulted in the formation of a separate phase and made the xerogel opaque. 4-fluorothiophenol was chosen as a capping agent, ${ }^{28}$ because it allows the dispersing of a high concentration of CdS NPs in $\mathrm{THF}^{29}$

\section{Preparation of doped ureasilicate xerogels}

The solution of the ureasilcate precursor was obtained by mixing stoichiometric $(1: 2)$ amounts of vacuum-dried Jeffamine ED-600 ${ }^{\circledR}(535 \mu \mathrm{L}, 0.936 \mathrm{mmol})$ and ICPTES (464 $\mu \mathrm{L}, 1.87 \mathrm{mmol}$ ) in a closed glass vessel, being stirred at approximately $200 \mathrm{rpm}$ for 10 minutes. The reaction was accompanied by an exothermic effect and resulted in the formation of a viscous ureasilicate precursor. The $\mathrm{pH}$ value of the ureasilicate precursor measured with "Merck" indicator paper was adjusted from 7.5 to 4.5 by addition of small excess of ICPTES (100 mL, $0.404 \mathrm{mmol})$. TEOS (250 mL, $1.12 \mathrm{mmol})$ was added establishing the molar ratio Jeffamine/ICPTES/TEOS equal to 1: 2: 1.2 . Then $1.0 \mathrm{~mL}$ of the solution of CdS nanoparticles in THF was added dropwise to the precursor.

The mixture was poured into a teflon mould with an inner diameter of $42 \mathrm{~mm}$. The mould was maintained in a confinement chamber with saturated water/ammonia vapours, obtained from a concentrated ammonium hydroxide solution $(25 \% \mathrm{w} / \mathrm{w})$ at room temperature. Under these conditions, the formation of a dense gel was observed 
within 6 hours, but the end of the process of solidification was completed after 2 days and was indicated by the ungluing of the xerogel from the mould walls.

The samples, labelled as w4, w6 and w8, corresponding to different sizes of CdS NPs in THF with $w$ values equal to $4,6,8$ respectively were produced in this manner. Then they were kept in an oven at $40^{\circ} \mathrm{C}$ for 2 days allowing evaporation of the residual liquids and showing approximately $7 \%$ shrinkage in diameter, their final thickness being about $0.45 \mathrm{~mm}$. An undoped xerogel labelled U(600)TEOS was synthesized following the same procedure.

The transparent ureasilicate nanocomposites prepared at different $w$ values are shown in Fig. 1a. The flexibility of the samples (Fig. 1b) is supposed to be ensured by the soft polymeric segments covalently bonded with siloxane backbone.

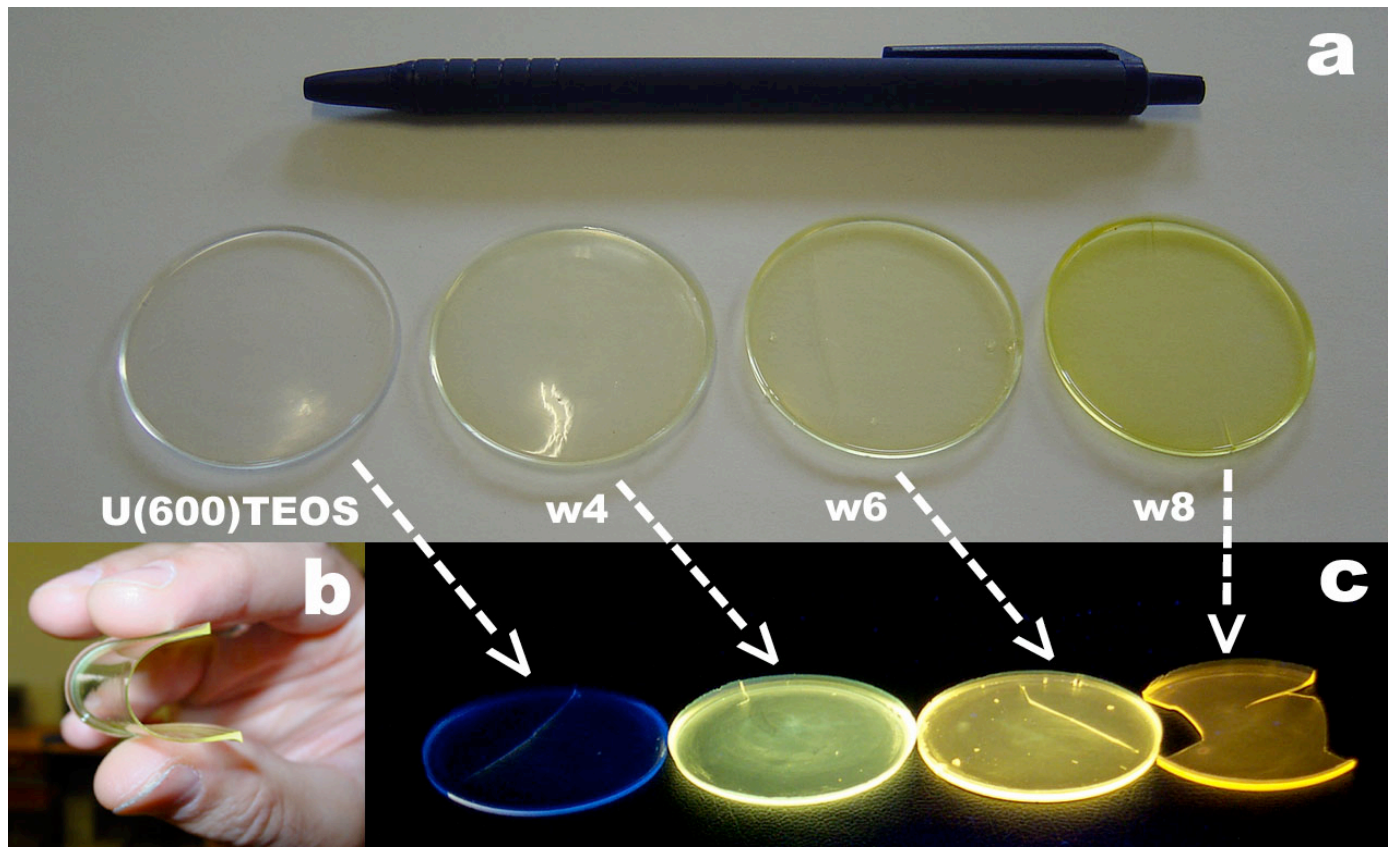

Figure 1. a) Pure and doped ureasilicate matrices b) Demonstration of sample flexibility: CdS doped nanocomposite $(\mathrm{w}=8)$ c) The PL response of the pure matrix (U(600)TEOS) and of the matrices (w4, w6, w8), doped with CdS NPs with different sizes (excitation wavelength of $365 \mathrm{~nm}$ ). 


\section{Characterization}

Optical properties of CdS colloidal solutions and ureasilicate nanocomposites were analyzed at visible range by optical absorption spectroscopy by Shimadzu UV-2501 PC spectrophotometer and photoluminescence (PL) steady state measurements by Spex Fluorolog spectrophotometer at front face geometry $(1.0 \mathrm{~mm}$ slit width, $1.7 \mathrm{~nm}$ band path) at room temperature. The obtained spectra were corrected by the emission correction factor of the detection system.

The cadmium and sulphur contents in the ureasilicate nanocomposites were measured by inductively coupled plasma optical emission spectroscopy (ICP-OES) by taking the average value of four independent measurements. The nanocomposites were digested in a microwave oven by using $10 \mathrm{~mL}$ mixture of concentrated nitric acid, hydrochloric acid and fluoric acid with a ratio of $1 / 3 / 0.2 \mathrm{~mL}$.

The surface of the CdS doped ureasilicates was qualitatively analyzed for the presence of cadmium and sulphur ions by time-of-flight secondary ion mass spectroscopy (TOF-SIMS) in static mode. This method has a good sensitivity and high mass resolution making it very convenient for analysis of small quantities of elements. The TOF-SIMS spectra were collected from $500 \times 500 \mu \mathrm{m}^{2}$ of the analyzed sample area at final impact energy of an ion beam of $25 \mathrm{keV}$, positive ion detection and primary beam intensity of $1.3 \mathrm{pA}$. The primary ions were generated by a Ga source.

Infrared spectra of CdS nanoparticles and doped ureasilicates were obtained at room temperature in the $4000-625 \mathrm{~cm}^{-1}$ range by averaging 25 scans, with resolution less then $1 \mathrm{~cm}^{-1}$ (Bomem MB104). Solid samples were prepared by pressing them into $\mathrm{KBr}$ pellets.

The influence of heating on the optical properties of undoped ureasilicate was examined by thermal annealing at $95^{\circ} \mathrm{C}$ in a dry atmosphere. 


\section{Results and discussion}

Water is necessary for hydrolysis and condensation of the ureasilicate precursor. However, CdS NPs capped with 4-fluorothiophenol molecules aggregates in water. ${ }^{28}$ Consequently, the direct addition of water into the ureasilicate precursor containing $\mathrm{CdS}$ NPs would provoke their spontaneous aggregation. Because of this, the gelation procedure was performed by exposing the reaction mixture to water/ammonia vapours. A similar technique was used to improve the mechanical properties of silicaantireflecting coatings ${ }^{30}$ and for the formation of glass from silica colloid solutions. ${ }^{31}$

The Jeffamine molecules, which are long polyether chains with doubleterminated amino groups, easily associate water molecules. FTIR spectrum of Jeffamine, kept for several days at ambient humidity and room temperature, indicates an appearance of a broad band around $3500 \mathrm{~cm}^{-1}$ and a relatively weak peak at $1646 \mathrm{~cm}^{-1}$ assigned to $\mathrm{O}-\mathrm{H}$ stretching and deformation (bending) vibrational modes. Most probably, the water is preferably adsorbed on the surface of the polymer due to the formation of $\mathrm{H}-\mathrm{O}-\mathrm{H} \ldots \mathrm{N}$ hydrogen bonds. Then water molecules penetrate into the volume along the hydrophilic oxygen species of the polyether chains. This assumption should be valid for the ureasilicate precursor as well, taking into account the fact that the final product, obtained by the reaction between silicon modified alkoxide and amino terminated block polymers, leads to the formation of urea (-NHCONH-) linkages. ${ }^{32}$ This reaction is based on the interaction between lone pair electrons of the amino group (electron donor) and an electron-poor carbon atom sandwiched right between two electronegative atoms in the isocyanate group.

Water, needed to convert alkoxy silane groups to silanol groups by hydrolysis, is supplied by the vapours of the saturated ammonia aqueous solution. Then, the silanol 
groups are deprotonated by the $\mathrm{NH}_{3}$ catalyst, giving nucleophilic $\mathrm{SiO}^{-}$species, which attack other neutral silanol groups, resulting in the formation of -Si-O-Si- bonds by a polycondensation reaction. ${ }^{33}$ The repetition of these processes with silica species, covalently bonded to the polyether chains, leads to the formation of a three dimensional organic-inorganic network at room temperature (Fig. 2).

Incorporation of TEOS causes the variation of the organic/inorganic ratio of the final hybrid material and changes their mechanical and thermal properties. ${ }^{34}$ Increase in TEOS concentration leads to the formation of a more rigid and compact material. However, the optically clear materials can be obtained, if the molar ratio between TEOS and Jeffamine is not greater than 1.2. The silica species, obtained by hydrolysis and condensation of TEOS most probably fit in the siliceous network, taking part in the formation of the structure shown schematically in Fig. 2. At a higher TEOS content, the final material becomes opaque, which may be explained by the appearance of a separate silica phase.

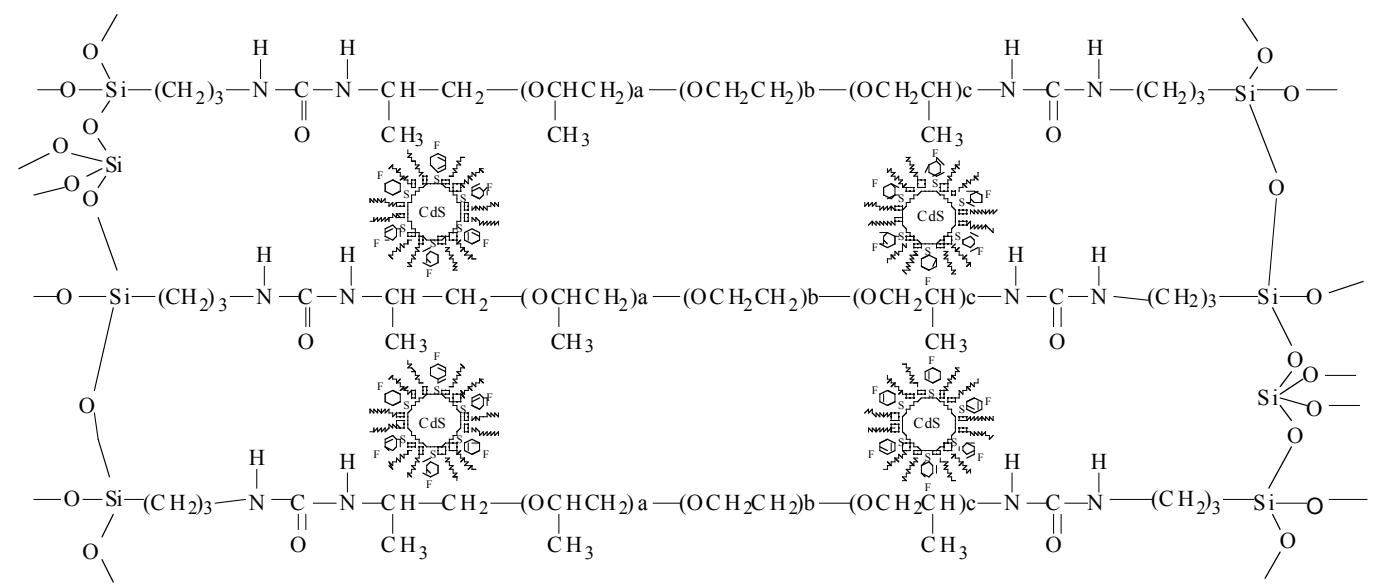

Figure 2. Schematic representation of CdS doped ureasilicate nanocomposite.

Omm - AOT molecule, $\mathrm{S}\langle\overline{\langle}\rangle \mathrm{F}$ - ligand 
Optimisation of the preparation conditions is very important for producing transparent, optically clear ureasilicates. Long term heating during sol-gel process (up to 3 weeks) at temperatures around $80^{\circ} \mathrm{C}$ under atmospheric conditions, ${ }^{31,35}$ resulted in the change of colour of the material from colourless to pale yellow. Obviously, this process would screen the optical response of CdS nanoparticles, immobilized in the ureasililicate and has to be minimized. It seems that a bathochromic shift observed in a pure U(600)TEOS xerogel correlates with changes in optical transparency of the pure liquid Jeffamine after annealing at ambient conditions (Fig. 3).

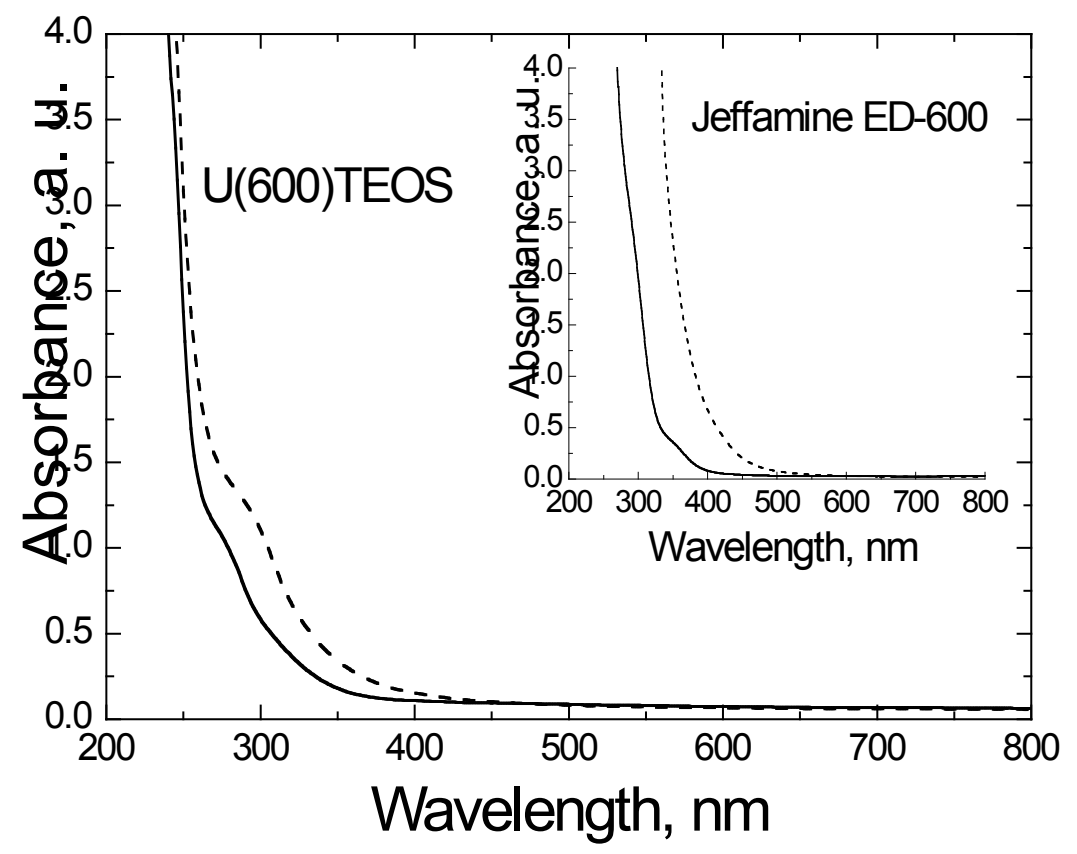

Figure 3. Change in the absorption edge of U(600)TEOS after annealing in a dry atmosphere and at $95^{\circ} \mathrm{C}$ for 10 days. Solid and dashed lines show the initial and afterannealing position of the absorption edge respectively. The change in the absorption edge for pure Jeffamine ED-600 annealed in a dry atmosphere and at $95^{\circ} \mathrm{C}$ for 4 days is given on the inset. 
Similar results have been reported, ${ }^{36}$ although the change of colour occurred much faster in comparison with the present study. Therefore, the technological procedure used for the preparation of samples strongly affects their index of yellowness. The mechanism of the degradation process has not been investigated in detail, but it is supposed that the residual amount of non-reacted amino groups and water molecules remaining in the ureasilicates deteriorates the optical stability of the material with heating. In the described preparation procedure an over-stoichiometric amount of ICPTES neutralizes the residual amino groups, resulting in improvement of the optical stability of the final xerogels. A moderate regime of drying, used in this work also contributes to the fabrication of a transparent solid xerogel, obtained after a complete evaporation of the THF.

The control over the starting $\mathrm{pH}$ of the ureasilicate precursor is another important condition for the preparation of the optically clear CdS nanocomposites. It was established that the CdS colloidal solution in THF readily flocculates, if a small amount of Jeffamine is added. Jeffamine has a basic nature ( $\mathrm{pH}$ around 8.5$)$ due to the presence of lone pair electrons of nitrogen in the amino groups. At higher $\mathrm{pH}$ value, a cleavage of the S-Cd bonds can happen, followed by the removal of the stabilizing ligands, attached to the surface of the NPs. The reason for the cleavage may be similar to the process of deprotonation of thiols in alkaline media.

The reaction of Jeffamine with ICPTES at stoichiometric ratio (1:2) leads to a decrease of the basicity of the medium down to 7.5. In fact, the formed amide groups are non-basic since the non-bonding electrons of nitrogen are delocalized by resonance with carbonyl groups. However, this decrease in $\mathrm{pH}$ is not enough to make the colloidal solution of CdS NPs stable. In order to neutralize some residual non-reacted amino groups, an additional amount of ICPTES must be added. It was experimentally found 
that decreasing of $\mathrm{pH}$ down to 4.5, ensures the stability of the NPs in the ureasilicate precursor.

It must be pointed out that CdS original colloidal solution in THF flocculated during 1 hour, when exposed to ammonia vapours. This did not happen, when these solutions was preliminary added to the ureasilicate liquid sol. It can be supposed that ureasilicate precursor wrap NPs protecting them against direct interaction with ammonia vapours.

Cadmium and sulphur contents in the doped ureasilicates obtained by ICP-OES are presented in Table 1. The excess of sulphur can be explained by the FPhS-groups, capping the surface of the NPs. For all investigated samples, the amount of cadmium and sulphur was in the range between 0.1 and 0.2 of weight percentage with respect to the matrix.

Table I. Cadmium and sulphur content in CdS-doped ureasilicates.

\begin{tabular}{|c|c|c|}
\hline Sample & Cd, weight \% & S, weight \% \\
\hline W4 & 0.10 & 0.13 \\
\hline W6 & 0.13 & 0.19 \\
\hline W8 & 0.16 & 0.20 \\
\hline
\end{tabular}

The FTIR spectrum of a CdS powder, obtained after evaporation of THF, and the FTIR spectrum of an ureasilicate nanocomposite, doped with CdS NPs, are shown in Figure 4. The first spectrum demonstrates the absence of S-H stretching vibration located at $2560 \mathrm{~cm}^{-1}$ in the pure $\mathrm{FPhSH}$, which is in agreement with the result, reported by Hosokawa et al. ${ }^{28}$ The absence of this peak may be explained by the cleavage of the $\mathrm{S}-\mathrm{H}$ bonds due to the interaction of lone pair electrons of sulfur with the Cd-rich surface of NPs. This process allows the collection of CdS powder from the reverse micellar 
solution, because of the change of the NPs' surface from hydrophilic to hydrophobic. Peaks at $1590 \mathrm{~cm}^{-1}$ and $1490 \mathrm{~cm}^{-1}$ correspond to $-\mathrm{C}=\mathrm{C}$ - stretching of an aromatic ring (aromatic skeletal modes). The well defined peak at $1227 \mathrm{~cm}^{-1}$ is attributed to C-F stretching vibration, and the peak at $818 \mathrm{~cm}^{-1}$ is related to the two adjacent hydrogens on the aromatic ring. These signals are attributed to the fluorophenyl ligands, capping the surface of the NPs.

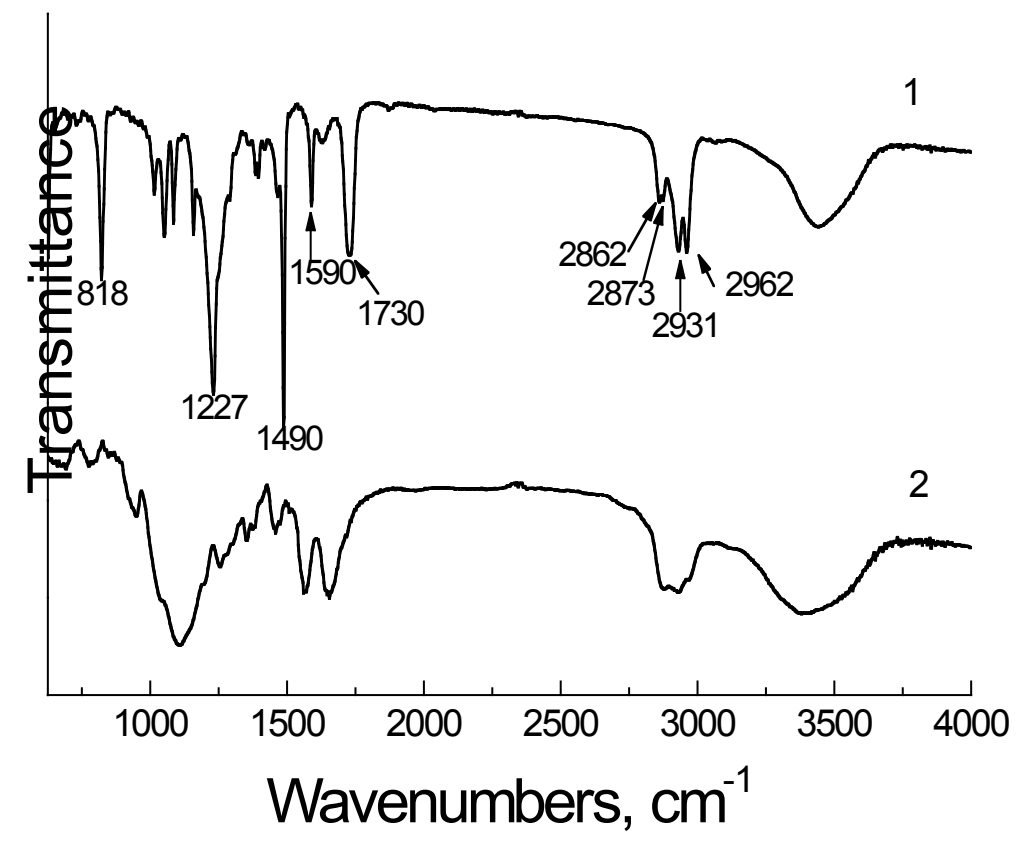

Figure 4. FTIR spectra of FPhS-/AOT-protected CdS NPs (1) and CdS-doped nanocomposite (2).

The peaks at $2962 \mathrm{~cm}^{-1}$ and $2873 \mathrm{~cm}^{-1}$ are assigned to asymmetric $\left(v_{\mathrm{a}}\left(\mathrm{CH}_{3}\right)\right)$ and symmetric $\left(v_{\mathrm{s}}\left(\mathrm{CH}_{3}\right)\right)$ stretching vibrations of methyl groups, the peaks at $2931 \mathrm{~cm}^{-1}$ and $2862 \mathrm{~cm}^{-1}$ are assigned to asymmetric $\left(\mathrm{v}_{\mathrm{a}}\left(\mathrm{CH}_{2}\right)\right)$ and symmetric stretching $\left(\mathrm{v}_{\mathrm{s}}\left(\mathrm{CH}_{2}\right)\right)$ vibrations of methylene groups respectively. The peak at $1730 \mathrm{~cm}^{-1}$ is attributed to a carbonyl stretching vibration $\left(v_{\mathrm{s}}(\mathrm{CO})\right)$. All these signals coincide with characteristic peaks of AOT molecules used for stabilization of the NPs in inverse micelles. 
From the FTIR analysis, it seems reasonable to conclude that CdS NPs are capped not only by FPhS-groups, but also surrounded with residual AOT molecules, remaining after the washing procedure. They might be attached to the NPs' surface by an ionic interaction of the sulfonate groups with the cadmium ions ${ }^{37}$ and might additionally protect them against aggregation. In fact, the pale yellow wax-like powder, obtained after evaporation of isooctane from the micellar solution containing CdS NPs, but without adding $\mathrm{FPhSH}$, was completely soluble in non-polar solvents, such as hexane, isooctane, THF, giving a transparent solution. This observation confirms the results reported for CdSe-containing microemulsions. ${ }^{38}$ This suggests that the polar headgroups of the surfactant molecules surrounding the water pools in the microemulsion are still bound to the surface of the NPs after the drying procedure and preserve them against degradation in the powder form.

Taking into account the alternative character of the poly(ethylene)/poly(propylene) (hydrophobic) and oxygens (hydrophilic) units, making up the organic chains in the ureasilicate structure, the FPhS-/AOT protected NPs are most likely localized in the proximity of the hydrophobic poly(ethylene)/ poly(propylene) units (see Fig. 2).

The FTIR spectrum of the nanocomposite practically coincides with the spectrum of undoped U(600)TEOS. The comprehensive study of the similar system in mid-infrared region was reported by Bermudez et al. $^{32}$. The absence of bands characteristic of the NPs is most probably due to their relatively low content in the matrix. This excludes their identification by FTIR.

In order to confirm the presence of the capped $\mathrm{CdS}$ in the finial nanocomposites, a TOF-SIMS study was performed. This surface-sensitive technique was earlier successfully used for identification of low concentrations of contaminants in organic 
and biological materials. Fig. 5 shows the positive static TOF-SIMS spectrum of the CdS-doped ureasilicate nanocomposite, labelled as w8. The spectral lines corresponding to ${ }^{114} \mathrm{Cd}^{+}$and to all its isotopes are clearly seen in this figure. Fig. 6 also shows the presence of $\mathrm{CF}^{+}, \mathrm{CF}_{3}^{+}$and $\mathrm{C}_{3} \mathrm{~F}_{3}^{+}$, which can originate from fluorobenzen ligands capping the NPs. The signal corresponding to $\mathrm{Na}^{+}$can come from residual AOT molecules. Signals, related to other organic radicals are probably due to the fragmentation of hydrocarbon parts of AOT molecules and polyoxy(ethylene)/polyoxy(propylene) chains. The signal from the sulphur is not presented in Figure 5, because it was near the detection level of the equipment. The fact is that the relative yield of sulphur to the TOF-SIMS spectra is much lower than that of cadmium, especially at positive polarity, used in the present investigation. 

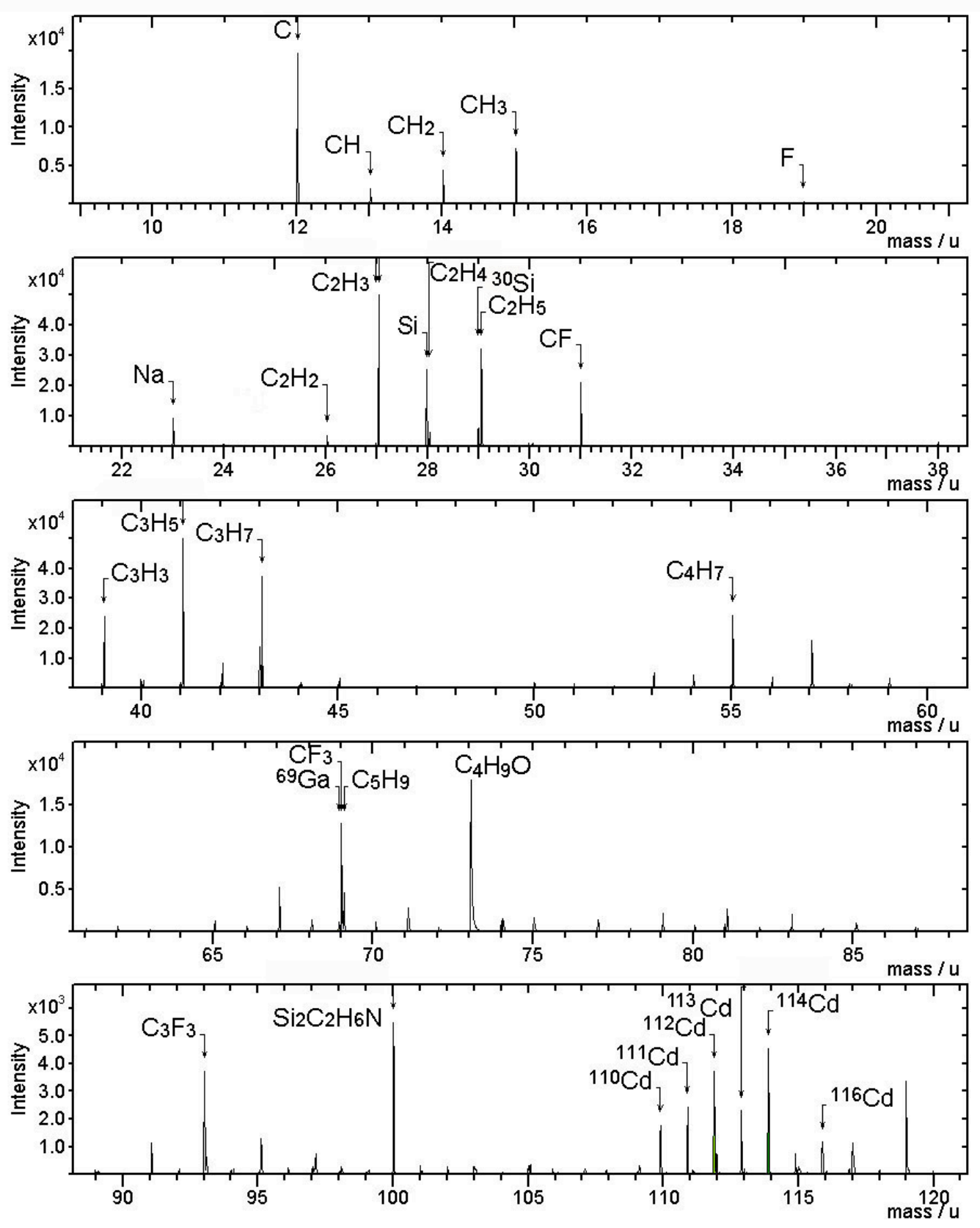

Figure 5. TOF-SIMS spectrum of CdS doped ureasilicate.

Fig.6 (a, b) shows the optical absorption and PL emission spectra of the doped and undoped nanocomposites and, for comparison, the spectra of the original CdS colloidal solutions used for the synthesis. As the original solutions, two doped samples $(w=4$ and $w=6)$ display a well defined absorption onset and absorption peak positions, 
due to the first excitonic transition. They shift towards shorter wavelengths with the decrease of $w$, i.e. with the decrease of the NPs size.

The missing of the maximum on the absorption curve of the sample with $w=8$ did not permit us to define the exact excitonic peak position, but the shift of the absorption onset to the shorter wavelength compared to that of the bulk CdS unambiguously proves the presence of quantum size effect for this sample as well. A decrease of the oscillator strength with increasing particle size is typical for CdS clusters ${ }^{39,40}$ and it is believed to be caused by surface trapped electron-hole pairs ${ }^{41}$.

The particle size in the colloidal solutions and CdS-doped ureasils presented in Table 2 was found from the position of the exciton resonance energy state, using a sizegap correlation curve based on tight-binding calculations. ${ }^{42}$ In the case of $w=8$, when there was no maximum on the absorption spectrum, this position was estimated by a second derivative plot of the curve. 


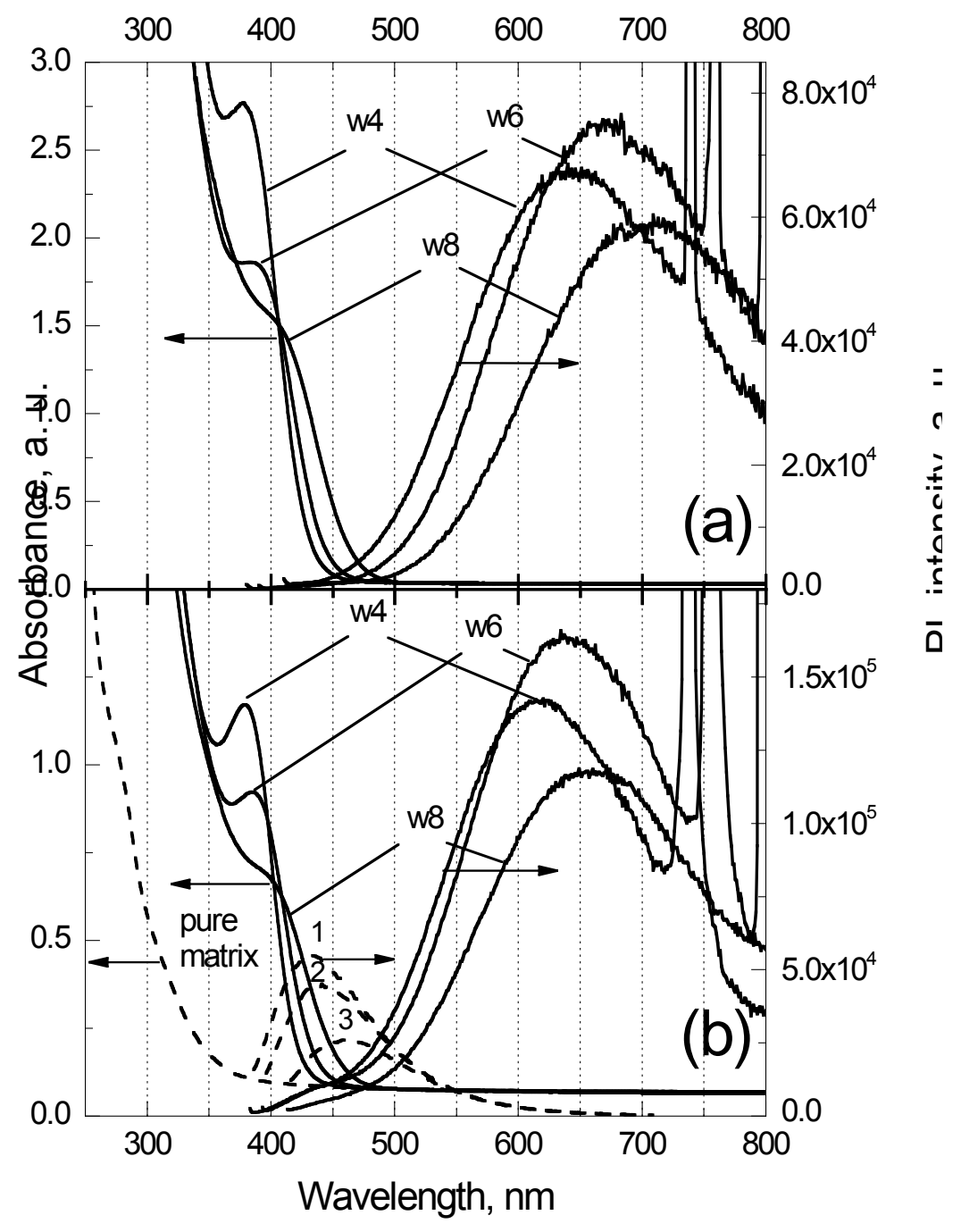

Figure 6. Absorbance and PL curves for CdS NPs in the original solutions (a) and for doped urreasilicate xerogels with $w=4, w=6$ and $w=8$ (b). Dashed curves show the absorbance of an undoped matrix and its PL spectra at different excitation wavelength $(1-375 \mathrm{~nm}, 2-385 \mathrm{~nm}, 3-410 \mathrm{~nm})$.

In the low energy range the scattering contribution to the absorbance is relatively small, which confirms the visible transparency of the samples.

The excitation wavelengths $(375,385$ and $410 \mathrm{~nm})$, used for PL measurements were selected to correspond to the absorption band of the NPs. The PL emission of the 
pure matrix and the emission of the matrix doped with NPs slightly overlap in the range between 400 and $500 \mathrm{~nm}$, which shows up as a small bump in the spectra of the nanocomposites. The emission in pure ureasilicate was suggested to be associated with photo-induced proton transfer between $\mathrm{NH}_{2}{ }^{+} / \mathrm{N}^{-}$defects and radiative recombination, originating from siliceous nanodomains. ${ }^{43}$ The decrease of PL intensity of the CdSdoped nanocomposites, as compared to the pure ureasilicates in this region, can be explained by self-absorption of light, emitted from the matrix by the semiconductor NPs. The PL response for the pure matrix and for the samples with different $w$ (excitation wavelength of $365 \mathrm{~nm}$ ) is demonstrated in Fig.1c.

The values of Stokes' shift, PL peak position and PL FWHM for initial solutions and final xerogels are summarized in Table 2. The observed broad (FWHM about 200 $\mathrm{nm})$ PL emission of the NPs in the range between 500 and $800 \mathrm{~nm}$ is significantly red shifted (by 250-300 nm) with respect to the absorption peak. This PL behaviour is usually observed for the CdS NPs, capped with thiol ligands ${ }^{24,28,44}$ in contrast to CdS nanocrystals, synthesised in a hot mixture of surfactant with noncoordinated solvent ${ }^{45}$, where the PL emission is mainly due to band to band transition with Stokes' shift and broadening (FWHM) about $20 \mathrm{~nm}$ and $15 \mathrm{~nm}$, respectively. Big broadening of PL spectra and large Stokes' shifts are attributed to trap emission from deep surface states. 
Table II. Absorption peak position, particle size, PL peak position, PL FWHM and Stokes' shift for 3 samples in the original solution and in the xerogel.

\begin{tabular}{|l|c|c|c|c|c|c|}
\hline \multicolumn{1}{|c|}{ Sample } & \multicolumn{2}{c|}{ W4 } & \multicolumn{2}{c|}{ W6 } & \multicolumn{2}{c|}{ W8* $^{*}$} \\
\hline & solution & xerogel & solution & xerogel & solution & xerogel \\
\hline $\begin{array}{l}\text { Absorption peak } \\
\text { position (nm) }\end{array}$ & 377 & 378 & 384 & 385 & 413 & 410 \\
\hline $\begin{array}{l}\text { Particle size (nm) } \\
\text { PL peak position (nm) }\end{array}$ & 1.99 & 2.01 & 2.10 & 2.12 & 2.68 & 2.61 \\
\hline PL FWHM (nm) & 197 & 183 & 195 & 189 & 291 & 262 \\
\hline Stokes' shift (nm) & 272 & 245 & 291 & 261 & 297 & 256 \\
\hline
\end{tabular}

* There are no peaks on the absorption curves for this sample; the wavelength corresponding to the excitonic transition energy was estimated by a position of the minimum of the second derivative of absorbance as a function of wavelength.

The Stokes' shift and PL peak broadening decrease, when particles are transferred form the solution into the ureasilicate matrix. This can be explained by ammonia-passivation of their surface. It is known that amines ${ }^{46}$ or ammonia ${ }^{47}$ could be effective passivating agents for surface defects in CdS NPs. The presence of basic nitrogen may cause removing of lower energy traps sites inside band gap, which leads to the blue shift of the corresponding PL spectra ${ }^{46}$.

A slight decrease in wavelength of the absorption band onset has been observed for the CdS NPs after their incorporation into the matrix, the excitonic transition energy remaining practically unchanged for the samples $w 4$ and $w 6$. In the case of the sample $w 8$, a more significant change in the excitonic transition energy was probably due to the uncertainty of its determination. The shift of the absorption onset may be due to the alternation of the energetic structure of NPs surface states. ${ }^{48}$

It is worth noting that the excitonic transition peaks in the CdS-doped xerogels becomes more pronounced after gelling under ammonia vapours. This effect may be connected with more efficient capping of the NPs due to absorption of ammonia 
molecules, which can influence the intrinsic broadening associated with crystalline and chemical defects.

\section{Conclusions}

The hybrid organic-inorganic ureasilicate materials with embedded CdS NPs have been synthesized. The experimental protocol has been developed to ensued NPs stability during the whole process of synthesis. The obtained nanocomposites demonstrated high transparency in visible range combined with flexibility, smoothness and long-term stability. After incorporation of CdS NPs into the ureasilicate matrix their optical properties were essentially preserved.

The presence of hydrophilic polyether chains in the ureasilicate precursor made it miscible in water rendering this material as a potential medium for the introduction of semiconductor NPs coated with stabilizing hydrophilic shells. The ureasililicates are compatible with organic protic or aprotic solvents allowing the incorporation of the NPs preliminarily dispersed in ethanol, propanol or THF.

The flexibility of the final xerogels can additionally extend the range of applications of these materials allowing them to be positioned on non-planar or curved surfaces.

\section{Acknowledgments}

This work has been supported by the Fundação para a Ciência e a Tecnologia (FCT). V.Boev and A.Soloviev thank the FCT for research grants SFRH/BD/3188/2000 and SFRH/BPD/18098/2004 respectively. The authors are indebted to Dr. Carmen Serra and Dr. Jorge Millos (CACTI-University of Vigo, Spain) for TOF-SIMS and ICP-OES measurements. 


\section{References}

[1] P. Horan, W. Blau, J. Chem. Phys., 92 (1990) 4139.

[2] N. Nirmal, C. B. Murray, M. G. Bawendi, Phys. Rev. B, 50 (1994) 2293.

[3] A. N. Goldstein, C. M. Echer, A. P. Alivisatos, Science, 256 (1992) 1425.

[4] R. Reisfeld, Sol-Gel Glasses and Modern Application, Springer-Verlag, 85 (1996) 99.

[5] N. Peyghambarian, E. Hanamura, S.W. Koch, Y. Masumoto, E.M. Wright, Nanostructured Materials: Synthesis, Characterization and Uses, ed. A.S. Edelstein, H. Hahan, Adam Higler, Bristol, (1993) 173.

[6] Y. Wang, A. Suna, W. Mahler, R. Kasowski, J. Chem. Phys., 87 (1987) 7315.

[7] K. Misawa, H. Yao, T. Hayashi, T. Kobayashi, Chem. Phys. Lett., 113 (1991) 183.

[8] Yi Yang, J. Huang, S. Liu, and J. Shen, J. Mater. Chem., 7 (1997) 131.

[9] Y. Wang, N. Herron, J. Phys. Chem., 91 (1987) 257.

[10] M. Nogami, K. Nagasaka, E. Kato, J. Am. Chem. Soc., 73 (1990) 2097.

[11] H. Minti, M. Eyal, R. Reisfeld, G. Berkovic, Chem. Phys. Lett., 183 (1991) 277.

[12] C. Li, M. Wilson, N. Haegel, J. Mackenzie, E. Knobbe, C. Porter, R. Reeves, Proc. SPIE, 1692 (1992) 326.

[13] W. Granier, L. Boudes, A. Pradel, M. Ribes, J. Allégre, G. Arnaud, P. Lefebvre, and H. Mathieu, J. Sol-Gel. Sci. Technol., 2 (1994) 765.

[14] H. Mathieu, T. Richard, J. Allègre, P. Lefebvre, G. Arnaud, J. Appl. Phys., 77 (1995) 287.

[15] V. Ptatschek, B. Schreder, K. Herz, U. Hilbert, W. Ossau, G. Schottner, O. Rahäuser, T. Bischof, G. Lermann, A, Materny, W. Kiefer, G. Bacher, A. Forchel, D. Su, M. Giersig, G. Müller, L. Spanhel, J. Phys. Chem., 101 (1997) 8898.

[16] A. Martucci, P. Innocenzi, J. Fick, J. D. Mackenzie, J. Non-Cryst. Solids, 204 (1999) 55 . 
[17] L. Spanhel, E. Arpac, H. Schmidt, J. Non-Cryst. Solids, 147\&148 (1992) 657.

[18] J. Lee, V. C. Sundar, J. R. Heine, M. G. Bawendi, K. F. Jensen, Adv. Mater., 12 (2000) 1102.

[19] A. C. C. Esteves, O. C. Montiero, A. M. Barros-Timmons, C. Boemare, M. J. Soares, T. Monteiro, T. Trinidade, J.Nanosci.Nonotech., 2 (2002) 177

[20] V. I. Boev, C. J. R. Silva, G. Hungeford, M. J. M. Gomes, J.Sol.Gel.Sci.Tech., 31 (2004) 131

[21] C. Bullen, P. Mulvaney, C. Sada, M. Ferrari, Al. Chiasera, Al. Martucci, J. Mat. Chem., 14 (2004) 1112.

[22] C. Li, N. Murase, Langmuir, 20 (2004) 1.

[23] T. Hirai, H. Okudo, I. Komasawa, J. Mat. Chem. 10 (2000) 2592.

[24] T. Gacoin, L. Malier, G. Counio, J.-P. Boilot, Proc. SPIE, 3136 (1997) 358.

[25] M. A. Correa-Duarte, M. Giersig, L. M. Liz-Marzán, Chem. Phys. Lett., 286 (1998) 497.

[26] M. Epifani, G. Leo, M. Lomascolo, L. Vasanelli, J. Sol-Gel Sci. Technol., 26 (2003) 441.

[27] S. Shiojiri, T. Hirai, I. Komasawa, J. Chem. Eng. Jap., 30 (1997) 86.

[28] H. Hosokawa, T. Ogata, Y. Wada, K. Murakoshi, T. Sakata, H. Mori, S. Yanagida, J. Chem. Soc., Faraday Trans., 22 (1996) 4575.

[29] T. Gacoin, L. Malier, J.-P. Boilot, Chem. Mat., 9 (1997) 1502.

[30] P. F. Belleville, H. G. Floch, Proc. SPIE, 2288 (1994) 25.

[31] G. W. Scherer, J. C. Luong, J. Non-Cryst. Solids, 6 (1984) 163.

[32] V. Z. Bermudez, L. D. Carlos, M. C. Duarte, M. M. Silva, C. J. R. Silva, M. J.

Smith, M. Assunção, L. Alcácer, J. Alloys and Compounds, 275-277 (1998) 21.

[33] R. K. Iller, The Chemistry of Silica, Wiley, New York, 1979. 
[34] P. Judeinstein, C. Sanchez, J. Mater. Chem., 6 (1996) 511.

[35] V. Z. Bermudez, L. D. Carlos, L. Alcácer, Chem. Mater., 11 (1999) 569.

[36] V. Boev, J. Pérez-Juste, I. Pastoriza-Santos, C. J. R. Silva, M. J. M. Gomes, L.M. Liz-Marzán, Langmuir, 20 (1999) 10268.

[37] T. Nakanishi, B. Ohtani, K. Uosaki, J. Phys. Chem. B, 102 (1998) 1571.

[38] M. L. Steigerwald, A. P. Alivisatos, J. M. Gibson, T. D. Harris, R. Kortan, A. J. Muller, A. M. Thayer, T. M. Duncan, D. C. Douglass, L. E. Brus, J. Am. Chem. Soc., 110 (1988) 3046.

[39] Y. Wang, A. Suna, J. McHugh, E. F. Hilinski, P.A. Lucas, R. D. Johnson, J. Chem. Phys., 92 (1990) 6927.

[40] L. E. Brus, J. Chem. Phys., 80 (1984) 4403.

[41] Y. Wang, N. Herron, J. Phys. Chem., 95 (1991) 525.

[42] Y. Wang, N. Herron, Phys. Rev. B, 42 (1990) 7253

[43] L. D. Carlos, R. A. Sá Ferreira, R. N. Pereira, M. Assunção, V. Z. Bermudez, J.Phys. Chem. 108 (2004) 14924 and references herein.

[44] N. Herron, Y. Wang, H. Eckert, J. Am. Chem. Soc., 112 (1990) 1322.

[45] W. W. Yu, X. Peng, Angew. Chem., 114 (2002) 2474.

[46] T. Dannhauser, M. O’Neil, K. Johansson, D. Whitten, G. McLendon, J. Phys. Chem., 90 (1986) 6074.

[47] Y. Wang, N. Herron, W. Mahler, A. Suna, J. Opt. Soc. Am. B, 6 (1989) 808. [48] T.Tsuruoka, K. Akamatsu, H. Nawafune, Langmuir, 20 (2004) 11169. 